\title{
Approximate solution of a fractional order model of HCV infection with drug therapy effect.
}

\author{
S.Z. Rida ${ }^{1}$, A.S. Abdel Rady ${ }^{1}$ A.A.M. Arafa ${ }^{*}$, and M. Khalil ${ }^{2}$ \\ ${ }^{1}$ Department of mathematics, Faculty of Science, \\ South Valley University, Qena, Egypt \\ Email: szagloul@yahoo.com, safwat_ahmed@hotmail.com, anaszi2@yahoo.com \\ ${ }^{2}$ Department of mathematics, Faculty of Engineering, \\ Modern Science and Arts University (MSA), Giza, Egypt \\ Email: m_kh1512@yahoo.com
}

\begin{abstract}
In this paper, we investigate the accuracy of the Generalized Euler method (GEM) for solving the fractional order model of HCV infection numerically. The fractional derivatives are described in the Caputo sense. We will study on the effect of treatment in blocking virion production and reducing new infections throughout the presented numerical simulation.
\end{abstract}

Keywords: Generalized Euler method, Fractional order ordinary differential equations, Models of infectious diseases.

\section{$1 \quad$ Introduction}

Nearly 170 million people worldwide are infected with hepatitis C virus (HCV) [1]. Chronic HCV infection is a major cause of cirrhosis necessitating liver transplantation and often leading to death [11]. Egypt has the highest rate of infections by hepatitis $\mathrm{C}$ virus (HCV) genotype 4 [8]. The current treatment for $\mathrm{HCV}$ infection involves combination therapy with two drugs, pegylated interferon (PEG-IFN) and ribavirin (RBV) [6]. Combination therapy with pegylated interferon and ribavirin, the latest treatment for $\mathrm{HCV}$ infection, elicits long-term responses in only about $50 \%$ of patients treated. No effective alternative treatments exist for non-responders. Consequently, significant efforts are continuing to maximize response to combination therapy. The responses of 
patients to combination therapy fall into three broad categories [6]. Sustained virological response (SVR) occurs when the concentration of viral particles in the blood, called viral load, falls below the detection limit ( 100 HCV RNA copies per $\mathrm{ml}$ ) during therapy and remains undetectable for 24 weeks after cessation of therapy [10]. Patients exhibiting SVR are generally cured of the infection. In some patients, the viral load becomes undetectable during therapy, called an end-oftreatment response (ETR), but relapses upon cessation of therapy. Here we present a fractional order generalization of a basic HCV model including the immune response (Ir) term proposed in [1]. Neumann et al [17] adapted the basic model of viral dynamics to HCV. The model assumes a simplified view of HCV infection and describes the response to interferon therapy through the coupled evolution of three populations, the uninfected hepatocytes, the productively infected hepatocytes, and the free HCV virions with the following equations

$$
\begin{aligned}
& \frac{d T}{d t}=s-d T-(1-\eta) \beta V T, \\
& \frac{d I}{d t}=(1-\eta) \beta V T-\delta I, \\
& \frac{d V}{d t}=\left(1-\varepsilon_{p}\right) p I-c V .
\end{aligned}
$$

Where $T$ represents uninfected hepatocytes, $I$ represents infected hepatocytes and $V$ represents virus. The model assumes that uninfected hepatocytes are produced at a constant rate $s$, die at rate $\mathrm{d}$ per cell and are infected at constant rate $\beta$. Infected hepatocytes are lost at a rate $\delta$ per cell. Viral particles (virions) are produced at rate $p$ per infected hepatocytes and cleared at rate $\mathrm{c}$ per virion. The efficacy of treatment in blocking virion production and reducing new infections are described by two parameters $\varepsilon_{p}$ and $\eta$ respectively. Ahmed and El-Saka [1] developed the system (1) by adding a new term to the second equation of (1) and introduced fractional-order into the model of HCV infection.

The new system is described by the following set of FDEs of order $\alpha_{1}, \alpha_{1}, \alpha_{3}$ where $0<\alpha_{1}, \alpha_{1}, \alpha_{3} \leq 1$ :

$$
\begin{aligned}
& D^{\alpha_{1}}(T)=s-d T-(1-\eta) \beta V T \\
& D^{\alpha_{2}}(I)=(1-\eta) \beta V T-\delta I\left(1-I / c_{2}\right), \\
& D^{\alpha_{3}}(V)=\left(1-\varepsilon_{p}\right) p I-c V .
\end{aligned}
$$

With $T(0)=2.3908 \times 10^{6}, I(0)=2.06897 \times 10^{6}, V(0)=10^{6}$

This term added in equation (1) represents the well-known high and low zone tolerance of the immune response (There is no Ir if the antigen number is either too high or too low [10]) since it vanishes for both limits $I \rightarrow 0$, and $I \rightarrow c_{2}$. Immune response is significant for long time virus dynamics. The system (2) 
assumes a simplified view of HCV infection and describes the response to interferon therapy through the coupled evolution of three populations, uninfected hepatocytes, productively infected hepatocytes, and free HCV virions.

No effective alternative treatments exist for non-responders. Consequently, significant efforts are continuing to maximize response to combination therapy. Chronic HCV infection is treated using interferon-a in combination with the antiviral drug ribavirin. Interferon acts primarily by blocking the production/release of new virus, although we also allow for a treatment effect in blocking de novo infection. Ribavirin alone induces either a transient early decline or no decrease in HCV viral load, but in combination with interferon it significantly improves long-term response rates. Here we present a model of HCV dynamics in which, on the basis of growing evidence, we assume that ribavirin decreases HCV infectivity in an infected individual in a dose-dependent manner [7]. The efficacy of treatment in blocking virion production and reducing new infections are described by two parameters $\varepsilon_{p}$ and $\eta$ respectively.

The rest of the paper is organized as follows. A brief review of the fractional calculus theory is given in Section 2. In section 3 a discussion about generalized Taylor formula is presented, while section 4 represents generalization of Euler method. Section 5 is devoted for the numerical results of the HCV model.

\section{Fractional calculus}

Fractional order ordinary differential equations, as generalizations of classical integer order ordinary differential equations, are increasingly used to model problems in fluid flow, mechanics, viscoelasticity, biology, physics and engineering, and other applications [5,14]. Fractional derivatives provide an excellent instrument for the description of memory and hereditary properties of various materials and processes. The differential equations with fractional order [12] have recently proved to be valuable tools to the modeling of many physical phenomena. This is because of the fact that the realistic modeling of a physical phenomenon does not depend only on the instant time, but also on the history of the previous time which can also be successfully achieved by using fractional calculus $[13,15]$.

In general, there exists no method that yields an exact solution for fractional differential equations [9]. Only approximate solutions can be derived using linearization or perturbation methods. Several methods have been suggested to solve fractional differential equations like the homotopy analysis method (HAM) [15], Adomian's decomposition method (ADM) [12].

Definition 1. The fractional integral of order $\alpha>0$ of a function $f: R^{+} \rightarrow R$ is given by

$$
\begin{aligned}
& J^{\alpha} f(x)=\frac{1}{\Gamma(\alpha)} \int_{0}^{x}(x-t)^{\alpha-1} f(t) d t, \quad \alpha>0, x>0 \\
& J^{0} f(x)=f(x) .
\end{aligned}
$$


Hence we have

$$
J^{\alpha} t^{\gamma}=\frac{\Gamma(\gamma+1)}{\Gamma(\alpha+\gamma+1)} t^{\alpha+\gamma}, \quad \alpha>0, \gamma>-1, t>0
$$

Definition 2. Riemann-Liouville and Caputo fractional derivatives of order $\alpha$ of a continuous function $f: R^{+} \rightarrow R$ is given respectively by

$$
\begin{aligned}
& D_{*}^{\alpha} f(x)=D^{m}\left(J^{m-\alpha} f(x)\right), \\
& D^{\alpha} f(x)=J^{m-\alpha}\left(D^{m} f(x)\right),
\end{aligned}
$$

Where

$$
m-1<\alpha \leq m, m \in N
$$

The definition of fractional derivative involves an integration which is non-local operator (as it is defined on an interval) so fractional derivative is a non-local operator. In other word, calculating time-fractional derivative of a function $f(t)$ at some time $t=t_{1}$ requires all the previous history, i.e. all $f(t)$ from $t=0$ to $t=$ $t_{1}$. So the reason of using fractional order differential equations (FOD) is that FOD are naturally related to systems with memory which exists in most biological systems. Also they are closely related to fractals which are abundant in biological systems. The results derived of the fractional system (2) are of a more general nature.

\section{Generalized Taylor's formula}

In this section we introduce a generalization of Taylor's formula that involves Caputo fractional derivatives. This generalization is presented in [19].

Suppose that $D_{*}^{k \alpha} f(x) \in C(0, a]$, for $k=0,1, \ldots, n+1$, where $0<\alpha \leq 1$. Then we have

$f(x)=\sum_{i=0}^{n} \frac{x^{i \alpha}}{\Gamma(i \alpha+1)}\left(D_{*}^{i \alpha}\right)(0+)+\frac{\left(D_{*}^{(n+1) \alpha} f\right)(\xi)}{\Gamma((n+1) \alpha+1)} x^{(n+1) \alpha}$

With $0 \leq \xi \leq x, \forall x \in(0, a]$.

In case of $\alpha=1$, the generalized Taylor's formula (3) reduces to the classical Taylor's formula. 


\section{Generalized Euler method (GEM)}

Most nonlinear fractional differential equations do not have analytic solutions, so approximations and numerical techniques must be used [18]. The decomposition method (ADM) and the variational iteration method (VIM) are relatively new approaches to provide an analytical approximate solution to linear and nonlinear problems, and they are particularly valuable as tools for scientists and applied mathematicians, because they provide immediate and visible symbolic terms of analytic solutions, as well as numerical approximate solutions to both linear and nonlinear differential equations. In recent years, the application of the ADM, VIM, in linear and nonlinear problems has been developed. On the other hand, these methods are effective for small time, i.e. $t<<1$, however the standard homotopy perturbation method (HPM) cannot solve the problem for larger time and in fact the solution of the chaotic system using HPM is an open problem [16]. Nevertheless by chance, there are cases at which these methods give good approximation for a large range of time $t$ [16]. A few numerical methods for fractional differential equations have been presented in the literature [14]. However many of these methods are used for very specific types of differential equations, often just linear equations or even smaller classes. In [18], Odibat and Momani derived the generalized Euler's method that we have developed for the numerical solution of initial value problems with Caputo derivatives. The method is a generalization of the classical Euler's method [1,2]. Consider the initial value problem

$D_{*}^{\alpha} y(t)=f(t, y(t)), y(0)=y_{0}, 0<\alpha \leq 1, t$

Let $[0, a]$ be the interval over which we want to find the solution of the problem (6). In actuality, we will not find a function $y(t)$ that satisfies the initial value problem (6). Instead, a set of points $\left\{\left(t_{j}, y\left(t_{j}\right)\right\}\right.$ is generated, and the points are used for our approximation. For convenience we subdivide the interval $[0, a]$ into $k$ subintervals $\left[t_{j}, t_{j+1}\right]$ of equal width $h=a / k$ by using the nodes $t_{j}=j h$, for $j$ $=0,1, \ldots, k$. Assume that $y(t), D_{*}^{\alpha} y(t)$, and $D_{*}^{2 \alpha} y(t)$ are continuous on $[0, a]$ and use the generalized Taylor's formula (5) to expand $y(t)$ about $t=t_{0}=0$. For each value $t$ there is a value $c_{1}$ so that

$y(t)=y\left(t_{0}\right)+\left(D_{*}^{\alpha} y(t)\right)\left(t_{0}\right) \frac{t^{\alpha}}{\Gamma(\alpha+1)}+\left(D_{*}^{2 \alpha} y(t)\right)\left(c_{1}\right) \frac{t^{2 \alpha}}{\Gamma(2 \alpha+1)}$

When $\left(D_{*}^{\alpha} y(t)\right)\left(t_{0}\right)=f\left(t_{0}, y\left(t_{0}\right)\right)$ and $h=t_{1}$ are substituted into equation (7), the result is an expression for $y\left(t_{1}\right)$ :

$y\left(t_{1}\right)=y\left(t_{0}\right)+f\left(t_{0}, y\left(t_{0}\right)\right) \frac{h^{\alpha}}{\Gamma(\alpha+1)}+\left(D_{*}^{2 \alpha} y(t)\right)\left(c_{1}\right) \frac{h^{2 \alpha}}{\Gamma(2 \alpha+1)}$ 
If the step size $h$ is chosen small enough, then we may neglect the second-order term (involving $h^{2 \alpha}$ ) and get

$y\left(t_{1}\right)=y\left(t_{0}\right)+\frac{h^{\alpha}}{\Gamma(\alpha+1)} f\left(t_{0}, y\left(t_{0}\right)\right)$

The process is repeated and generates a sequence of points that approximates the solution $y(t)$. The general formula for generalized Euler's method (GEM) when $t_{j+1}=t_{j}+h$ is

$y\left(t_{j+1}\right)=y\left(t_{j}\right)+\frac{h^{\alpha}}{\Gamma(\alpha+1)} f\left(t_{j}, y\left(t_{j}\right)\right)$

for $j=0,1, \ldots, k-1$. It is clear that if $\alpha=1$, then the generalized Euler's method (8) reduces to the classical Euler's method.

\section{$5 \quad$ Numerical results and discussion}

Two possible mechanisms of interferon action are assumed. Interferon could block de novo infection of cells with effectiveness $\eta$ or lower viral production from infected cells with effectiveness $\varepsilon_{p}$. In this section we will study the effect of $\eta$ and $\varepsilon_{p}$ on the dynamics of the HCV infection. By setting $s=2.6 \times 10^{4}, d=$ $0.0026, \beta=2.25 \times 10^{-7}, \delta=0.26,5 \times 10^{4} c_{2}=5 \times 10^{6}, c=6, p=0.2$.

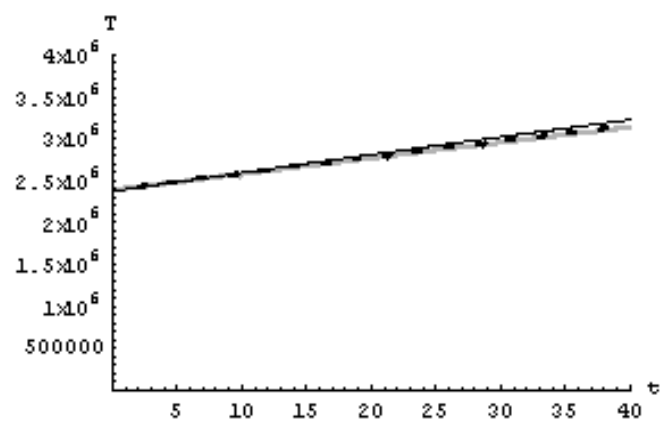

(a)

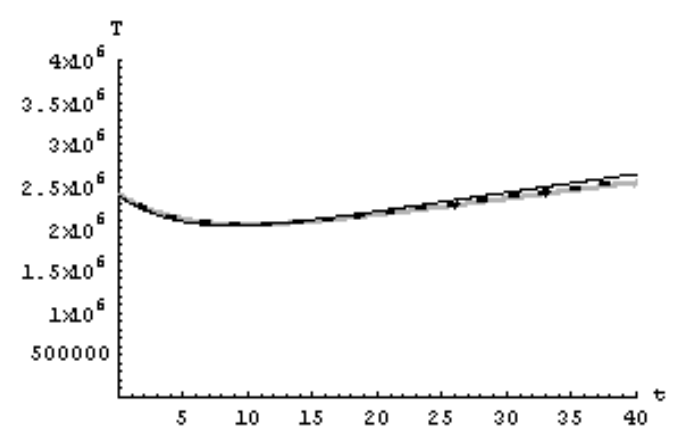

(b)

Fig.1. The density of uninfected hepatocytes $T$, for $\varepsilon_{p}=0.99, \eta=0.8(a)$, and $\varepsilon_{p}=0, \eta=0.8(b)$ : Gray solid line $(\alpha=1)$, Dotted line $(\alpha=0.99)$, Black solid line $(\alpha=0.95)$. 


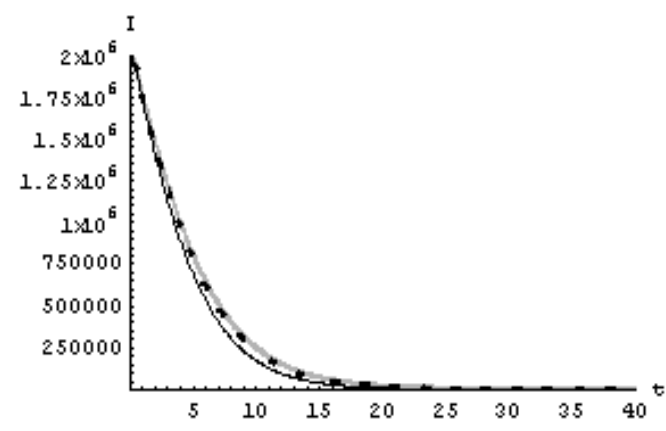

(a)

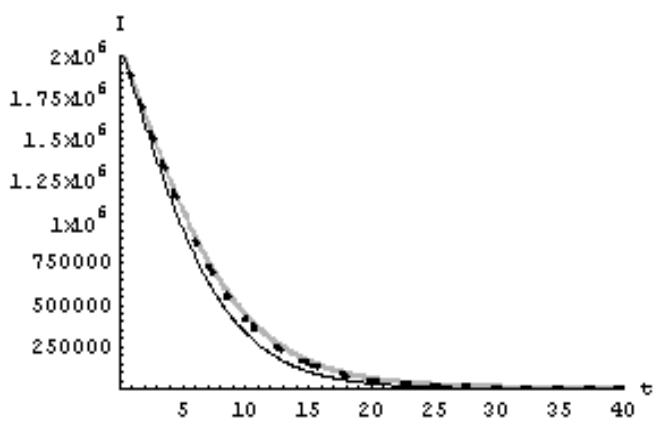

(b)

Fig.2. The density of infected hepatocytes $I$, for $\varepsilon_{p}=0.99, \eta=0.8(a)$, and $\varepsilon_{p}=0, \eta=0.8(b)$ : Gray solid line $(\alpha=1)$, Dotted line $(\alpha=0.99)$, Black solid line $(\alpha=0.95)$.

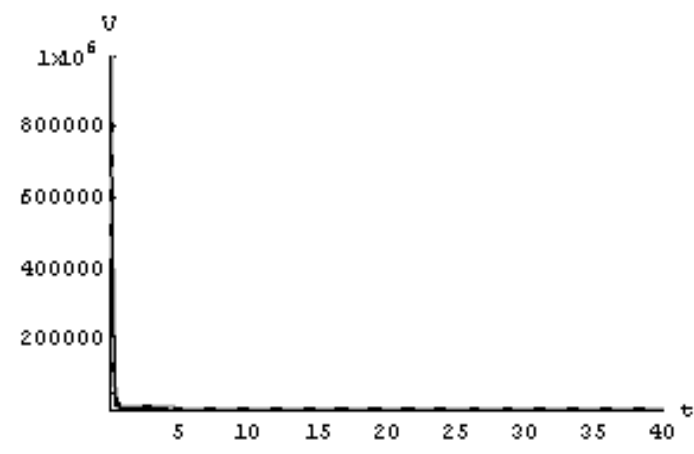

(a)

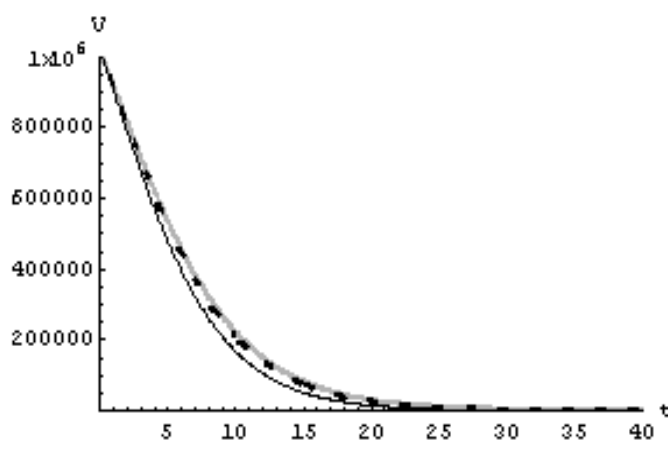

(b)

Fig.3. The population of the free viruses $V$, for $\varepsilon_{p}=0.99, \eta=0.8(a)$, and $\varepsilon_{p}=0, \eta=0.8(b)$ : Gray solid line $(\alpha=1)$, Dotted line $(\alpha=0.99)$, Black solid line $(\alpha=0.95)$.

\section{Conclusion}

In this paper, generalized Euler method (GEM) was implemented to describe the effect of the efficacy of treatment in blocking the HCV virion production and reducing new infections that are described by two parameters, $\varepsilon_{p}$ and $\eta$, respectively. Before treatment, $\eta=\varepsilon_{p}=0$ and a steady state exists where viral production is balanced by viral clearance and the production of infected cells is balanced by their loss. Uninfected hepatocytes are also in steady state determined by the balance between their production, death, and loss due to infection. Following the onset of therapy, at time $t=0, I$ and $V$ decline and $T$ increases due to 
drug action. Interestingly, $V$ decays exponentially with time if $\varepsilon_{p}=0$ and $\eta>0$ (Fig. 3.a), whereas if $0<\varepsilon_{p}<1$ and $\eta>0$ the decay occurs rapidly and the efficacy of treatment in blocking virion production appears clearly in (Fig. 3.b).

As a definition of fractional calculus: $\lim _{\alpha \rightarrow 1} D^{\alpha} f(t)=D f(t)$ has been provided. In the presented problem, $V(t), I(t), T(t)$ have been obtained. The results obtained show that when $\alpha \rightarrow 1$, the solution of the fractional model (2) $V_{\alpha}(t)$, $I_{\alpha}(t), T_{\alpha}(t)$, reduce to the standard solution $V(t), I(t), T(t)$. Fractional order results show the realistic biphasic decline behavior of HCV but at a slower rate. Since multi-drugs are strongly recommended to avoid drug resistance it is proposed that a further drug should be used in addition to Peg-IFN and RBV. Notice that RBV may not be considered as independent drug since, when used alone, it has no effect on $\mathrm{HCV}$.

\section{References}

[1] E. Ahmed, H.A. El-Saka, On fractional order models for Hepatitis C, Nonlinear Biomedical Physics, 4(2010) 4:1.

[2] A.A.M. Arafa, S.Z. Rida and M. Khalil, Fractional modeling dynamics of HIV and $C D 4^{+} T$-cells during primary infection, Nonlinear Biomedical Physics 6( 2012) 1-7.

[3] A.A.M. Arafa, S.Z. Rida and M. Khalil, Fractional Order Model of Human T-cell Lymphotropic Virus I (HTLV-I) Infection of $C D 4^{+} T$-cells, Advanced Studies in Biology, 3(2011) 347 - 353.

[4] Brunetto M, Colombatto P, Bonino F: Bio-mathematical models of viral dynamics to tailor antiviral therapy in chronic viral hepatitis. World $\mathbf{J}$ Gastroenterol 15(2009), 531-537.

[5] K.S. Cole, Electric conductance of biological systems, in: Proc. Cold Spring Harbor Symp. Quant. Biol, Cold Spring Harbor, New York, (1993) 107-116.

[6] Dahari H, Lo A, Ribeiro RM, Perelson AS: Modeling hepatitis C virus dynamics: Liver regeneration and critical drug efficacy. J Theoret Biol 247 (2007),371-381.

[7] Dahari H, Shudo E, Ribeiro RM, Perelson AS: Mathematical modeling of HCV infection and treatment. Hepatitis $\mathrm{C}$ methods and Protocols Humana Press, NJTang H , 2( 2008), 439-453.

[8] Das, P., Mukherjee, D. and Sarakar, J., Analysis of a disease transmission model of hepatitis C, Mathematical Biosciences and Engineering, 13(2005), 331-333.

[9] K. Diethelm and G. Walz, Numerical solution for fractional differential equations by extrapolation, Numerical algorithms, 16 (1997) 231-253. 
[10]Dixit N, Layden-Almer J, Layden T, Perelson AS: Modeling how ribavirin improves interferon response rates in hepatitis $\mathrm{C}$ virus infection. Nature 432 (2004), 922-924.

[11]Dusheiko, G., Schmilovitz,W.H., Brown, D., Mc Omish, F., Yap, P.L. and Simmonds, P., Hepatitis C virus genotypes: an investigation of type-specific differences in geographic origin and disease, Hepatology, 19(1994), 13-18.

[12]A.M.A. El-Sayed, S.Z. Rida, A.A.M. Arafa, On the Solutions of Timefractional Bacterial Chemotaxis in a Diffusion Gradient Chamber, International Journal of Nonlinear Science , 7(2009) 485-492.

[13]A.M.A. El-Sayed, S.Z. Rida, A.A.M. Arafa, Exact Solutions of FractionalOrder Biological Population Model, Commun. Theor. Phys. 52 (2009), 992996.

[14]A.M.A. El-Sayed, A. E. M. El-Mesiry, and H. A. A. El-Saka, Numerical solution for multi-term fractional (arbitrary) orders differential equations, Comput. Appl. Math., 23(2004)33-54.

[15]I. Hashim, O. Abdulaziz, S. Momani, Homotopy analysis method for fractional IVPs, Communications in Nonlinear Science and Numerical Simulation 14(2009), 674-684.

[16]O.D. Makinde, Adomian decomposition approach to a SIR epidemic model with constant vaccination strategy, App. Math. comput., 184 (2007), 842848.

[17]Neumann AU, Lam NP, Dahari H, Gretch DR,Wiley TE, et al. Hepatitis C viral dynamics in vivo and the antiviral efficacy of interferon- $\alpha$ therapy. Science 282(1998), 103-107.

[18]Zaid M. Odibat, and Shaher Moamni, An algorithm for the numerical solution of differential equations of fractional order, J. Appl. Math. \& Informatics, 26(2008), $15-27$.

[19]Z. Odibat and N. Shawagfeh, Generalized Taylor's formula, Appl. Math. Comput. 186 (2007), 286-293. 